\title{
Treatment of Persistent Post-Traumatic Headache with the Anti-CGRP Receptor Antibody Erenumab: A Case Report
}

Mike Papenhoff ( $\nabla$ mike.papenhoff@bg-klinikum-duisburg.de)

BG Klinikum Duisburg https://orcid.org/0000-0002-2128-4263

Marcel Dudda

Universitaetsklinikum Essen

\section{Research Article}

Keywords: Migraine-like posttraumatic headache, CGRP, erenumab, amitriptyline, restless legs, medication overuse

Posted Date: November 1st, 2021

DOI: https://doi.org/10.21203/rs.3.rs-267455/v2

License: (1) This work is licensed under a Creative Commons Attribution 4.0 International License.

Read Full License 


\section{Abstract}

Introduction: Calcitonin gene-related peptide (CGRP) has only recently emerged as a potential target in posttraumatic headache. To our knowledge this is the first description of a patient with persistent posttraumatic headache following severe traumatic head injury who has been treated with the CGRPreceptor-monoclonal antibody erenumab.

Case presentation: A 56-year-old man presented with a 30-year history of frequent migraine-like posttraumatic headaches following a high-voltage head injury that he treated with an excessive intake of peripheral analgesics. Although amitriptyline was reasonably effective, it had to be discontinued when the patient developed restless legs syndrome in the second year of treatment. Under therapy with erenumab he achieved a rapid and stable reduction in his symptoms down to only $2-3$ headache days per month without adverse events.

Conclusions: This case demonstrates that the inhibition of the CGRP-receptor with erenumab could be an effective treatment option in migraine-like post-traumatic headache even decades after the traumatic event.

\section{Introduction}

Severe post-traumatic headache can have serious effects on the individual patient's quality of life, particularly if it persists over a long period. However, the pathophysiological mechanisms of persistent post-traumatic headache remain elusive [1] and there have been no targeted treatment approaches until now. Thus, treatment is usually guided by the recommendations for primary headache disorders [2].

Erenumab is an anti-CGRP-receptor monoclonal antibody (CGRP = calcitonin gene-related peptide) and is effective in the prevention of episodic and chronic migraine $[3,4]$. Interestingly, recent preclinical studies suggest a role of CGRP in the development of post-traumatic headache $[5,6]$. This case describes a 56year-old man with a 30-year history of persistent migraine-like post-traumatic headache who achieved significant reduction of pain and a remarkable improvement of his quality of life under therapy with erenumab.

\section{Case Presentation}

A 56-year-old man presented with frequent, severe headache attacks that persisted since a high-voltage electrical injury 30 years ago. During a work-related accident in the 1980s, current from a railway overhead line $(15 \mathrm{kV})$ had entered through the right occipital region of the patient's head and exited through the left foot. During a 2-month hospital stay, he underwent multiple operations. These included having to surgically remove parts of the skull bone and reconstruct the scalp with an advancement flap.

The patient described a throbbing pain that emanated from the back of the head on the right and spread over the entire left side of the skull. He rated the pain intensity at 7-8 on a numerical rating scale (NRS 
$0-10)$. The attacks occurred on average at 12-13 days per month, lasted between 2 and 24 hours and were partly accompanied by nausea and vomiting. No visual aura was present.

Apart from two initial, ineffective rehabilitation procedures and an interim attempt at acupuncture, over the past decades pain management had merely consisted of medication with mainly peripheral analgesics as needed. It was only now that an attentive pharmacist, who had noticed the patient's extensive need for painkillers, had suggested an evaluation by a pain specialist. At this time, the patient reported that his intake of ibuprofen, a fixed combination of ASA + paracetamol + caffeine, metamizole and occasionally tramadol, amounted to more than 50 tablets each month (Table 1 ).

Clinical and neurological examination yielded normal results. Comorbid arterial hypertension was treated with lisinopril. Due to the frequent headaches, the patient had never been able to pursue a regular occupation and was prematurely retired aged 27 . He accepted only offers of marginal employment with a maximum workload of 4 days per month, working as a test driver. His results from psychometric tests (German pain questionnaire in 2007) were in the range of chronic pain patients but with a depression score on a high normal level.

We diagnosed a persistent, migraine-like post-traumatic headache. Despite the patient's extremely frequent use of peripheral analgesics, we considered a medication-overuse headache unlikely, since first, the pain location was strictly unilateral and second, the patient had experienced a sudden pain-free period during a two-week holiday which he managed to spend entirely without medication.

Figure 1 provides an overview over the patient's pain management. The patient started treatment with amitriptyline up to $20 \mathrm{mg}$ daily. He reported considerable relief especially in pain intensity and duration, which led to a substantial reduction in his analgesic medication (Table 1). He now was able to control the attacks solely with metamizole, which he used at 10 days/month (corresponding to 10 headache days/month). However, after 16 months of amitriptyline treatment, he developed restless leg syndrome (RLS). Since antidepressants are suspected to induce or worsen RLS [7], a switch to metoprolol was attempted. However, it was ineffective, the headaches markedly recurred and the patient resumed amitriptyline in combination with levodopa/benserazide. In this way he achieved satisfactory control of both headaches and RLS.

With additional intensive transcutaneous supraorbital nerve stimulation (t-SNS; Cefaly®, Cefaly Technology, Seraign, Belgium) the patient was able to discontinue amitriptyline after 2 years of treatment. He then reported intake of ibuprofen at 10 days/month (corresponding to 10 headache days/month) and levodopa/benserazide as needed every $4-5$ days.

The patient now inquired about erenumab as a treatment option. Treatment started 8 months later (after approval of erenumab and approval of cost reimbursement) with monthly subcutaneous injections of 70 $\mathrm{mg}$ erenumab. After the first injection, the number of headache days per month had already declined to 2-3 and has remained stable over the 9 months of treatment so far. The patient reported that the remaining headache attacks mainly occurred during the last days before the next erenumab injection was 
due and treatment with ibuprofen was sufficient. Pain intensity was rated at NRS 4-5. He also discontinued t-SNS application. The patient has not experienced adverse events so far and is extremely satisfied with the treatment. The RLS remained stable and is currently treated with dermal application of rotigotine. After the initiation of erenumab treatment the patient realized in retrospect that his social participation had been rather limited before and was now expanding. Amongst other activities, he had accepted more test-driving projects and had ventured on a holiday abroad.

Table 1

Use of analgesics and t-SNS

\begin{tabular}{|c|c|c|c|c|c|c|}
\hline & before pain management & & with amitript & line & $\begin{array}{l}\text { with } \\
\text { erenumab }\end{array}$ & \\
\hline \multirow{4}{*}{$\begin{array}{l}\text { Analgesic (intake } \\
\text { days per month) }\end{array}$} & ibuprofen (400-600 mg) & 20 & metamizole & 10 & ibuprofen & 3 \\
\hline & $\begin{array}{l}\text { ASA + paracetamol + caffeine } \\
(250 / 200 / 50 \mathrm{mg})\end{array}$ & 20 & & & & \\
\hline & metamizole (500-750mg) & 10 & & & & \\
\hline & tramadol (CR, 50mg) & $\begin{array}{l}2- \\
3\end{array}$ & & & & \\
\hline t-SNS use & no & & yes & & no & \\
\hline
\end{tabular}

\section{Conclusions}

This patient with persistent, migraine-like post-traumatic headache only received structured pain management after 30 years of treatment with analgesics as needed. Unfortunately, this is not uncommon, as post-traumatic headache remains an entity that is challenging to treat [8]. Often, the referral to a pain management specialist constitutes a hurdle that prevents patients from receiving optimal treatment. Only now, an attentive pharmacist who noticed the patients extensive need of painkillers, suggested an evaluation by a pain specialist.

Although amitriptyline treatment was effective and the patient was able to drastically reduce his extensive use of analgesics, it transpired not to be the optimal treatment when the patient developed RLS. Although it remains unclear whether the RLS was actually induced by amitriptyline or was a late effect of the injury, discontinuation of treatment was advisable due to a substantial risk of worsening the RLS symptoms [7].

Erenumab rapidly and effectively reduced the number of headache days per month to 2-3, without the need for additional t-SNS application. This was the best outcome the patient had experienced since his accident over 30 years ago and it substantially improved his psychosocial conditions and life circumstances. Only then did he realize that he had been rather limited by the constant fear of provoking headache attacks that had accompanied him through his entire adult life. 
Erenumab is a fully human monoclonal antibody directed specifically against the CGRP receptor. Unlike amitriptyline, it does not interfere with serotonin or histamine pathways and thus there is no reason for it to influence the existing RLS.

Regarding his 30-year pain history, we expect that this patient will require long-term erenumab treatment. The proven long-term efficacy and excellent safety profile over 5 years so far is thus reassuring [9]. Importantly, erenumab has a low interaction potential which is favourable considering the patient's comedication.

Despite a very similar phenotype, migraine-like post-traumatic headache and primary migraine were shown to be associated with differences in brain structure in a recent study based on magnetic resonance imaging suggesting differences in pathophysiology [10]. However, this does not exclude a common role for CGRP as both entities are supposedly multifactorial processes. Recent preclinical studies of mild traumatic brain injury, using rat models, showed that treatment with an anti-CGRP monoclonal antibody following concussion ameliorated pain-related behaviour and hypersensitivity in the acute phase [5,6]. However, delayed administration of the CGRP inhibitor appeared to be ineffective in preventing persistent post-traumatic headaches in rodents which led the authors to the conclusion that GCRP was mainly involved in the development of central sensitization after the event and was thus associated with promoting (but not sustaining) persistent post-traumatic headache [6].

In a recent report, impressive results were obtained with erenumab in 7 severely symptomatic patients with acute migraine-like post-traumatic headache secondary to mild traumatic head injury. The patients started treatment between 4 and 11 weeks after the trauma and all achieved $a \geq 95 \%$ reduction in headache days per month [11].

Furthermore, Ashina et al. conducted a single-arm, open label study with erenumab for the prevention of persistent PTH attributed to mild traumatic brain injury. During the 12-week treatment period, erenumab reduced the monthly number of headache days by 2.8 days among 89 patients (baseline: 15.7 mean headache days per month) [12].

Our case provides a first observation that erenumab can also be effective in persistent post-traumatic headache following severe traumatic head injury even decades after the onset of symptoms. Since persistent post-traumatic headache is a common health issue, robust controlled studies on anti-CGRPreceptor treatment should be undertaken.

Therefore, erenumab should be considered as a therapy, which seems to be safe, convenient, and low in side effects in the treatment of persistent post traumatic headaches with migraine-like phenotype.

\section{Abbreviations}

ASA acetylsalicylic acid 
NRS numerical rating scale

RLS restless leg syndrome

t-SNS transcutaneous supraorbital nerve stimulation

\section{Declarations}

\section{Acknowledgements}

The authors thank Rainer Lauterbach, Ph.D., of Novartis Pharma GmbH, Germany, and Sonja Hergeth, Ph.D. of medizinwelten-services $\mathrm{GmbH}$, Stuttgart, Germany, for providing medical writing/editorial support, which was funded by Novartis Pharma GmbH, Germany, in accordance with Good Publication Practice Guidelines.

\section{Funding}

Medical writing support was funded by Novartis Pharma GmbH. We acknowledge support by the Open Access Publication Fund of the University of Duisburg-Essen, Germany.

\section{Competing interests}

The authors declare that there is no conflict of interest.

\section{Ethics approval}

Ethics approval was not required since this report describes a case from routine clinical practice.

\section{Consent to participate}

Consent to participate was not required since this report describes a case from routine clinical practice.

\section{Consent for publication}

Written informed consent was obtained from the patient for publication of this case report

\section{Availability of data and material}

Not applicable.

\section{Code availability}

Not applicable.

\section{Authors' contributions}


MP: conception, drafting, final approval, agrees to be accountable for all aspects of the work in ensuring that questions related to the accuracy or integrity of any part of the work are appropriately investigated and resolved.

MD: funding acquisition, critically revising, and adding important intellectual content

MP and MD: All authors have read and agreed to the published version of the manuscript

\section{References}

1. Defrin R. Chronic post-traumatic headache: clinical findings and possible mechanisms. J Man Manip Ther. 2014; https://doi.org/10.1179/2042618613Y.0000000053

2. Larsen EL, Ashina H, Iljazi A, Al-Khazali HM, Seem K, Ashina M, Ashina S, Schytz HW. Acute and preventive pharmacological treatment of post-traumatic headache: a systematic review. J Headache Pain. 2019; https://doi.org/10.1186/s10194-019-1051-7

3. Goadsby PJ, Reuter U, Hallstrom Y, Broessner G, Bonner JH, Zhang F, Sapra S, Picard H, Mikol DD, Lenz RA. A Controlled Trial of Erenumab for Episodic Migraine. N Engl J Med. 2017; https://doi.org/10.1056/NEJMoa1705848

4. Tepper S, Ashina M, Reuter U, Brandes JL, Dolezil D, Silberstein S, Winner P, Leonardi D, Mikol D, Lenz R. Safety and efficacy of erenumab for preventive treatment of chronic migraine: a randomised, double-blind, placebo-controlled phase 2 trial. Lancet Neurol. 2017; https://doi.org/10.1016/S14744422(17)30083-2

5. Bree D, Levy D. Development of CGRP-dependent pain and headache related behaviours in a rat model of concussion: Implications for mechanisms of post-traumatic headache. Cephalalgia. 2018; https://doi.org/10.1177/0333102416681571

6. Navratilova E, Rau J, Oyarzo J, Tien J, Mackenzie K, Stratton J, Remeniuk B, Schwedt T, Anderson T, Dodick D, Porreca F. CGRP-dependent and independent mechanisms of acute and persistent posttraumatic headache following mild traumatic brain injury in mice. Cephalalgia. 2019; https://doi.org/10.1177/0333102419877662

7. Kolla BP, Mansukhani MP, Bostwick JM. The influence of antidepressants on restless legs syndrome and periodic limb movements: A systematic review. Sleep Med Rev. 2018; https://doi.org/10.1016/j.smrv.2017.06.002

8. Seifert TD, Evans RW. Posttraumatic headache: a review. Curr Pain Headache Rep. 2010; https://doi.org/10.1007/s11916-010-0117-7

9. Ashina M, Goadsby PJ, Reuter U, Silberstein S, Dodick DW, Xue F, Zhang F, Paiva da Silva Lima G, Cheng S, Mikol DD. Long-term efficacy and safety of erenumab in migraine prevention: Results from a 5-year, open-label treatment phase of a randomized clinical trial. European journal of neurology. 2021; https://doi.org/10.1111/ene.14715 
10. Schwedt TJ, Chong CD, Peplinski J, Ross K, Berisha V. Persistent post-traumatic headache vs. migraine: an MRI study demonstrating differences in brain structure. J Headache Pain. 2017; https://doi.org/10.1186/s10194-017-0796-0

11. Charles JA. Treatment of posttraumatic headache migraine phenotype with erenumab - An observational study. J Concussion. 2019; https://doi.org/10.1177/2059700219878292

12. Ashina H, lljazi A, Al-Khazali HM, Eigenbrodt AK, Larsen EL, Andersen AM, Hansen KJ, Brauner KB, Morch-Jessen T, Chaudhry B, Antic S, Christensen CE, Ashina M, Amin FM, Schytz HW. Efficacy, tolerability, and safety of erenumab for the preventive treatment of persistent post-traumatic headache attributed to mild traumatic brain injury: an open-label study. J Headache Pain. 2020; https://doi.org/10.1186/s10194-020-01136-z

\section{Figures}

\section{High-voltage head injury in 1985 (aged 25)}

Since then severe, frequent headache attacks

Past interventions: 2 ineffective rehabilitation procedures, analgesics as needed ( $\geq 20$ days/month)

Prematurely retired aged 27

\section{Symptoms and Findings:}

Left-sided, throbbing pain; NRS 7-8; partly with nausea/vomiting, $12 \mathrm{x} /$ month; duration $2-24 \mathrm{~h}$

Depression score in high normal range*

Normal clinical exams

Diagnosis: persistent, migraine-like PTH
Unsuccessful attempt to stop amitriptyline Ibuprofen at 10-15 days/month Start t-SNS application

\section{Symptoms and Findings:}

Patient reports restless legs $\rightarrow$ Treatment attempt with levodopa/benserazide + neurological examination

Diagnosis: RLS (amitriptyline induced/enforced?)

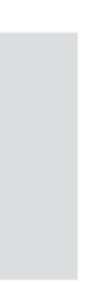

$07 / 2018$

Intensive t-SNS usage enables discontinuation of amitriptyline Ibuprofen at 10 days/month Levodopa/benserazide every $4 / 5$ days

\section{$05 / 2016$}

Amitriptyline $1 \mathrm{x}$ daily $10 \mathrm{mg}$

Patient education (reduction of analgesics, non-drug therapy options)

\section{$06 / 2016$}

With amitriptyline substantial pain relief. Analgesics reduced (metamizole $10 \mathrm{~d} /$ month)

\section{7/2017}

With amitriptyline + t-SNS good pain control

\section{$10 / 2017$}

RLS: levodopa/benserazide

PTH: switch to metoprolol unsuccessful.

Amitriptyline continued $+\mathrm{t}-\mathrm{SNS}$

\section{$03 / 2019$}

First injection with erenumab

\section{With erenumab}

Reduction to 2-3 headache days/month

Ibuprofen at 2-3 days/month

t-SNS application discontinued

Increasing social participation 


\section{Figure 1}

Timeline of the patient's pain management 